\title{
Review
}

\section{Review of Research on Routes of Helicobacter pylori Infection}

\author{
Hang Li \\ Department of Radiation Medicine, Tianjin Third Central Hospital, Tianjin China
}

\author{
Keywords \\ Helicobacter pylori, infection route, water-borne \\ spread \\ Correspondence \\ Hang Li, \\ E-mail: lihangtj@126.com
}

DOI: $10.1515 / i-2017-0105$

\begin{abstract}
In recent years, many scholars conducted in-depth research on Helicobacter pylori and identified it as an important pathogen of chronic gastritis and peptic ulcer. H. pylori also causes also and contributes to precancerous lesions (atrophic gastritis and intestinal metaplasia) and is closely related to occurrence and development of gastric adenocarcinoma and gastric mucosa-associated lymphoma. This study summarizes biological characteristics, epidemic status, and infection route of $H$. pylori and reviews research on roles of natural environments, especially drinking water, during infection.
\end{abstract}

In 1982, Marshall et al. ${ }^{[1]}$ observed Helicobacter pylori from gastric mucosal slices and discovered a potential etiological relationship between $\mathrm{H}$. pylori and gastric cancer. In recent years, many scholars performed in-depth research on $H$. pylori and identified this bacterium as important pathogen of chronic gastritis and peptic ulcer; $H$. pylori also causes and is a contributing factor to precancerous lesions (atrophic gastritis and intestinal metaplasia) and is closely related to occurrence and development of gastric adenocarcinoma and gastric mucosa-associated lymphoma ${ }^{[2]}$. In 1994, H. pylori was listed a class I carcinogen by the International Agency for Research on Cancer ${ }^{[3]}$. This study summarizes biological characteristics, epidemic status, and infection route of $H$. pylori and reviews research on roles of natural environments, especially drinking water, during infection.

\section{Biological characteristics}

H. pylori is a Gram-negative bacterium with a length of $2-4 \mu \mathrm{m}$ and width of $0.5-1 \mu \mathrm{m}$. This organism is also microaerophilic, suitably living at $34-40{ }^{\circ} \mathrm{C}, 5 \%-8 \% \mathrm{O}_{2}$, $5 \%-10 \% \mathrm{CO}_{2}$, and high-humidity environments. Although it can survive at $\mathrm{pH}<4$ acidic conditions, $H$. pylori can only reproduce at $\mathrm{pH}=5.5$ to $8.0^{[4]}$. H. pylori can adapt to acidic environments to a certain extent. In other words, in acidic environments, intracellular cytoplasmic $\mathrm{pH}$ value of $H$. pylori approaches neutral state. Thus, $H$. pylori remains capable of growth and reproduction ${ }^{[5]}$ in acidic environment of gastric juices.

H. pylori is generally present in suitable environments and is spherical in shape in in vitro culture, water and milk, and make contact with antibodies This spherical type may be a self-protecting state, posing difficulty in cultivating viable bacteria ${ }^{[4,6]}$.

\section{Epidemic status}

Human $H$. pylori infection is prevalent worldwide. However, infection rate varies widely across the world. In some developing countries, infection rate of $H$. pylori can reach up to $80 \%$, whereas in developed countries, such as the United States, infection rate totals $7.5 \%{ }^{[7-9]}$.

Pandeya et al. ${ }^{[10]}$ surveyed 1355 adults in Australian communities using serological methods to detect $H$. pylori and calculated an infection rate of $15.5 \%$. People born in Australia and New Zealand are less infected by this bacterium. Aje et al. ${ }^{[11]}$ conducted a study on 46 pairs of control group with indigestion in Nigeria. Results showed higher infection rate of $H$. pylori $(67.4 \%)$ in digestive population than that of the control group (78.3\%). However, causes of such results remain unclear. Dattoli et al. ${ }^{[12]}$ performed serological tests among 1104 children aged 4 to 11 years old in Salvador, Brazil and measured an infection rate of $H$. pylori of $28.7 \%$. Kesanos et al. ${ }^{[13]}$ researched on $H$. pylori infection among 101 Albanians and 101 Greeks in the Western Balkans and determined an infection rate of 54\% 
among Albanians; infection rate in Greeks reached 34\%. Epplein et al. ${ }^{[14]}$ performed a case-control study on 689 lowyield class African-Americans and observed an infection rate of as high as $79 \%$. Sonnenberg et al. ${ }^{[15]}$ used laboratory databases to analyze biopsy information of 78,985 Americans and measured $7.5 \%$ infection rate. Li et al. ${ }^{[16]}$ conducted a large-scale systematic research on gastrointestinal diseases in Shanghai and investigated 3153 people aged 18 to 80 years old and detected an infection rate of $73.3 \%$.

Childhood is a critical period of infection, and most $H$. pylori infections occur initially among children below 10 years old. Upon infection, $H$. pylori survives in the body for a long time and is rarely eradicated. Children of developed countries exhibit significantly lower infection rate than those in developing countries. In the United States, infection rate of children under 12 totals $10 \%$ to $15 \%$. In France, infection rate of children under 10 is $3.5 \%$, whereas in northern Nigeria and Zambia, children under five years present an infection rate of $50 \%$. In each province in mainland China, average infection rate of children measures $63.9 \%{ }^{[17]}$. Previous studies also showed relatively low infection rate among populations with good economic condition, good health condition, and good health habit ${ }^{[10-16,18]}$, implying possible patterns and routes of $H$. pylori infection.

\section{Infection routes of $\boldsymbol{H}$. pylori}

Infection routes of $H$. pylori remain unclarified. These routes may be human-human spread and environmental infection.

\section{Human-human spread}

Human-human spread results from direct contact with people and mainly occurs among families or acquaintances, such as parents and children, husband and wife, and brothers and sisters. Areas with good health conditions and low infection rate feature low probability of maternal-neonatal spread, whereas spread among siblings serves as the main route ${ }^{[19]}$. Cervantes et al. ${ }^{[20]}$ observed that $H$. pylori infection among siblings can be used as means of precaution for younger brothers and sisters, especially when age difference is less than three years old. Human-human spread can be divided into two specific forms, namely, oral-oral spread and fecal-oral spread.

Oral-oral spread Studies on oral-oral spread showed that $H$. pylori can survive in vomit for specific periods, even reaching up to $10 \%$ survival after $24 \mathrm{~h}$. As a result of vomiting or reflux, $H$. pylori can also subsist in saliva, gingiva, and plaque ${ }^{[21]}$, providing convenient conditions for oral-oral spread. Hongying et al. ${ }^{[22]}$ conducted a questionnaire survey and serological tests among 478 children in Kunming and performed logistic regression analysis [odds ratio (OR) $=2.260)$ and noted that shared tableware and shared toothbrush $(\mathrm{OR}=2.094)$ are independent risk factors for $H$. pylori infection. Kivi et al. ${ }^{[23]}$ investigated, sampled, and identified 104 subjects among 39 families to explore specific routes of spread among family members. Results showed that in addition to mother and child, siblings and spouse can also spread bacteria through shared use of daily necessities and kisses. Zou et al. ${ }^{[24]}$ performed meta-analysis on a previous study summary and discovered significantly higher infection rate of $H$. pylori (45.0\%) in mouths of people with $H$. pylori in the stomach than those without (23.9\%). With drug treatment, removal rate of $H$. pylori in the stomach can reach up to $85.8 \%$, whereas that of $H$. pylori in mouth reaches $5.7 \%$, indicating that infection and retention of $H$. pylori in the mouth possibly serve as important route of recurrent infection in the stomach.

Some studies do not support the above view. De Sousa et al. ${ }^{[25]}$ did not find $H$. pylori population in saliva and plaque compared with $H$. pylori population in the stomach. Vale et al. ${ }^{[26]}$ also found no evidence of spread among spouses.

Fecal-oral spread $H$. pylori can be cultured in secretions of final interception and rectum ${ }^{[27]}$; the bacterium is generally spherical in human feces and difficult to culture. However, polymerase chain reaction (PCR) can detect its DNA ${ }^{[28]}$, proving biological conditions for fecal-oral spread. Results of animal experiments support this route of spread. Cellini et al. ${ }^{\text {[29] }}$ fed 24 mice (12 infected and 12 uninfected mice) in a cage with separate compartments and fed another 24 mice (12 infected and 12 uninfected mice) in a cage without separate compartments. Results showed that mice in the cage without compartment were infected by fecal-oral spread. Oshio et al. ${ }^{[30]}$ conducted an animal experiment with gerbils and also witnessed such results. Laporte et al. ${ }^{[31]}$ conducted a cohort study in France and observed that teenagers with diarrhea in welfare houses can spread H. pylori, demonstrating fecaloral spread in populations.

Drinking water and food are the main intermediate media during fecal-oral spread. Although $H$. pylori is sensitive to chlorination disinfection, tap water with chlorination disinfection does not carry live bacteria. However, local water treatment and pipe networks present significant differences. Residual chlorine concentrations of terminal 
water differ, and secondary contamination may occur. The biofilm formed by microorganisms on tap water pipe wall may also protect $H$. pylori, allowing its survival in tap water. Thus, H. pylori are often detected in tap water. Tap water containing residual chlorine can still be used for culture of viable bacteria after 5 min chlorination disinfection, surviving organisms presenting spherical form even after $24 \mathrm{~h}^{[32]}$. In some areas with poor water treatment and poor sanitary condition, $H$. pylori is detected in tap water ${ }^{[33]}$ and is commonly found in some areas ${ }^{[34,35]}$. By contrast, $\mathrm{H}$. pylori can survive for shorter periods in milk ${ }^{[36]}$.

\section{Environmental infection}

Water, food, and other media containing H. pylori may cause H. pylori infection.

Water-borne infection $H$. pylori can survive in many water bodies. Cellini et al. ${ }^{[37]}$ and Queralt et al. ${ }^{[38]}$ detected $H$. pylori in marine waters, rivers, surface waters, and sewage. Viable bacteria are not easily cultured in the environment because of their spherical shape, but they can be detected by PCR. Cellini et al. ${ }^{[39]}$ reported that live bacteria can be cultured in seawater samples, probably because spiral rodshapes $H$. pylori can parasitize some microorganisms in seawater, allowing detection of this bacterium. Moreno et al. ${ }^{[40]}$ used improved membrane separation and enrichment techniques and fluorescence in situ hybridization to successfully culture viable bacteria, providing new evidence for environmental infection. Many studies on population epidemiology confirmed infection routes of H. pylori. Xiaoqin et al. ${ }^{[41]}$ investigated and detected 1127 subjects in Guizhou Province and used logistic regression and respectively observed significantly higher positive rates (64.2\% and 36.6\%) of anti-Hp-IgG and IgM of people drinking river water than those drinking tap water $(50.6 \%$ and $27.3 \%$ ) and well water (57.3\% and $28.1 \%$ ). Lihua et al. ${ }^{[42]}$ conducted investigations, sampling, and detection among 938 children and teenagers aged 3 to 18 years old in Gan $\mathrm{Su}$ and noted significantly higher infection rate of those drinking river water $(78.1 \%)$ and well water $(75.9 \%)$ than that those consuming tap water (68.1\%). Tianzhe et al. ${ }^{[43]}$ conducted a meta-analysis on previous research literature and discovered that drinking water habits are mostly correlated with $\mathrm{H}$. pylori infection. Drinking unboiled water $(\mathrm{OR}=3.08,95 \%$ confidence interval $(\mathrm{CI})=1.02-9.26)$ and unclean water $(\mathrm{OR}=2.01,95 \% \mathrm{CI}=1.35-3.00)$ is also an independent risk factor for infection.
Infection from food Few studies reported food infections. Papiez et al. ${ }^{[44]}$ observed that infection rate of $H$. pylori in shepherds reaches as high as $97.6 \%$ among Polish people. This value is much higher than that of the general population (65.1\%). Quaglia et al. ${ }^{[36]}$ indicated that $H$. pylori can survive in goat's milk for some time, and shepherds may be infected by drinking goat's milk. Some researchers reported that raw vegetables and meat can also increase risk of infection.

\section{Conclusion}

Various epidemiological studies showed that environmental mediators are important sources of $H$. pylori infection and feature significance to economic development and public health. However, no direct evidence shows that $H$. pylori can infect populations through environmental mediators. As a result of its biological characteristics, difficulty arises from culturing viable $H$. pylori in environmental samples. Results of PCR detection provide insufficient direct evidence. Thus, infection routes remain controversial. Recently, Moreno et al. ${ }^{[40]}$ cultured viable bacteria obtained in sewage. When this method is successfully used in other environmental samples, it will serve as an important culturing method for $\mathrm{H}$. pylori; although curative rate of $H$. pylori with drug treatment approximates $80 \%$ (slightly different according to strain virulence and drug resistance), recurrence rate is also high, and developing countries exhibit significantly higher yearly recurrence rate (13\%) than developed countries (2.67\%) ${ }^{[45-47]}$. In-depth studies covered the use of epidemiology, genomics, animal models, and other means to explore direct evidence of environmental infection routes of $\mathrm{H}$. pylori and to conduct risk assessment of environmental infection and pathogenicity; these research will provide scientific basis for development of public health policies that guide clinical treatment and intervening risk factors and solid foundation for effective prevention and treatment of $H$. pylori infection and diseases.

\section{Declarations}

\section{Acknowledgements}

No.

\section{Competing interests}

The author declare that he has no competing interest. 


\section{Authors' contributions}

$\mathrm{H} \mathrm{Li}$ made the literature analysis and wrote, discussed and revised the manuscript of this review.

\section{References}

1 Marshall BJ, Warren JR. Unidentified curved bacilli in the stomach of patients with gastritis and peptic ulceration. Lancet, 1984,16: Environ Health, December 2012, Vol.29, No.12 1311-1315.

2 Oliveira AG, Santos A, Guerra JB, et al. babA2- and cagA-positive Helicobacter pylori trains are associated with duodenal ulcer and gastric carcinoma in Brazil. J Clin Microbiol, 2003,41:3964-3966.

3 IARC Working Group on the Evaluation of Carcinogenic Risks to Humans. Schistosomes, liver flukes and Helicobacter pylori . IARC Monogr Eval Carcinog Risks Hum, 1994,61:1-241.

4 Kusters JG, van Vliet AH,Kuipers EJ. Pathogenesis of Helicobacter pylori infection. Clin Microbiol Rev, 2006,19:449-490.

5 Scott DR, Marcus EA, Wen Y, et al. Cytoplasmic histidine kinase (HP0244)-regulated assembly of urease with UreI,a channel for urea and its metabolites, $\mathrm{CO} 2, \mathrm{NH} 3$, and $\mathrm{NH} 4+$, is necessary for acid survival of Helicobacter pylori. J Bacteriol. 2010, 192:94-103.

6 Azevedo NF, Almeida C, Cerqueira L, et al . Coccoid form of Helicobacter pylori as a morphological manifestation of cell adaptation to the environment. Appl Environ Microbiol, 2007, 73:3423-3427.

7 Khalifa MM, Sharaf RR, Aziz RK. Helicobacter pylori: a poor man's gut pathogen. Gut Pathog, 2010, 2:2.

8 Goh KL, Chan WK, Shiota S, et al. Epidemiology of Helicobacter pylori infection and public health implications. Helicobacter, 2011, 16:1-9.

9 Malaty HM. Epidemiology of Helicobacter pylori infection. Best Pract Res Clin Gastroenterol, 2007, 21:205-14.

10 Pandeya N, Whiteman DC. Prevalence and determinants of Helicobacter pylori sero -positivity in the Australian adult community. J Gastroenterol Hepatol, 2011, 26:1283-1289.

11 Aje AO, Otegbayo JA, Odaibo GN, et al. Comparative study of stool antigen test and serology for Helicobacter pylori among Nigerian dyspeptic patients:a pilot study. Niger J Clin Pract, 2010,13:120-124.

12 Dattoli VC, Veiga RV, da Cunha SS, et al. Seroprevalence and potential risk factors for Helicobacter pylori infection in Brazilian children. Helicobacter, 2010,15:273-278.

13 Katsanos KH, Tatsioni A, Tsakiris V, et al. Helicobacter pylori is a major public health priority in western Balkans:an endoscopy referral center experience. Eur J Intern Med, 2010,21:306-309.

14 Epplein M, Signorello LB, Zheng W, et al. Race, African ancestry, and Helicobacter pylori infection in a low -income United States population. Cancer Epidemiol Biomarkers Prev, 2011,20:826-834.

15 Sonnenberg A, Lash RH, Genta RM. A national study of Helicobacter pylori infection in gastric biopsy specimens. Gastroenterology, 2010,139:1894-1901.

16 Li Z, Zou D, Ma X, et al. Epidemiology of peptic ulcer disease: endoscopic results of the systematic investigation of gastrointestinal disease in China. Am J Gastroenterol, 2010, 105:2570-2577.

17 Liu L, Zhang J. Helicobacter Pylori Infection in Children. Advances in Modern Biomedicine, 2008, 8 (11): 2132-2136.

18 Zhang Tianzhe, Zhang Tiemin, Zhao Dandan, etc. Meta-analysis of Helicobacter Pylori Infection in Chinese Population. World Chinese Journal of Digestology. 2009, 17 (15): 1582-1589.

19 Weyermann M, Rothenbacher D, Brenner H. Acquisition of Helicobacter pylori infection in early childhood: independent contributions of infected mothers,fathers, and siblings . Am J Gastroenterol, 2009, 104:182-189.

20 Cervantes DT, Fischbach LA, Goodman KJ, et al. Exposure to Helicobacter pylori: positive siblings and persistence of Helicobacter pylori infection in early childhood. J Pediatr Gastroenterol Nutr, 2010, 50:481-485.

21 Rasmussen LT, Labio RW, Gatti LL, et al. Helicobacter pylori detection in gastric biopsies,saliva and dental plaque of Brazilian dyspeptic patients. Mem Inst Oswaldo Cruz, 2010,105:326-330.

22 Mi Hongying, Li Li, Xu Xiaoyan. Analysis on Status and Risk Factors of Helicobacter Pylori Infection in Children in Wuhua District, Kunming. Chinese Journal of clinicians, 2011, 5 (18): 5465-5469.

23 Kivi M, Tindberg Y, Sorberg M,et al. Concordance of Helicobacter pylori strains within families. J Clin Microbiol, 2003,41:5604-5608.

24 Zou QH, Li RQ. Helicobacter pylori in the oral cavity and gastric mucosa:a meta-analysis. J Oral Pathol Med, 2011,40:317-324.

25 De Sousa L,Vásquez L,Velasco J,et al. Isolation of Helicobacter pylori in gastric mucosa,dental plaque and saliva in a population from the Venezuelan Andes. Invest Clin,2006,47:109-116.

26 Vale FF, Vítor JM. Genomic methylation:a tool for typing Helicobacter pylori isolates, Appl Environ Microbiol, 2007,73:4243-4249.

27 Kim do H, Jung HM, Hwang YJ, et al. Culture and polymerase chain reaction of Helicobacter pylori from rectal and terminal ileal fluid after polyethylene glycol (colyte)ingestion in healthy adults with positive urea breath test. Korean J Gastroenterol, 2010,56:27-32.

28 Falsafi T, Valizadeh N, Najafi M, et al. Culture of Helicobacter pylori from stool samples in children. Can J Microbiol, 2007, 53:411-416.

29 Cellini L, Dainelli B, Angelucci D, et al. Evidence for an oral-faecal transmission of Helicobacter pylori infection in an experimental murine model. APMIS, 1999,107:477-484.

30 Oshio I, Osaki T, Hanawa T, et al. Vertical Helicobacter pylori transmission from Mongolian gerbil mothers to pups. J Med Microbiol, 2009,58:656-662.

31 Laporte R, Pernes P, Pronnier P, et al. Acquisition of Helicobacter pylori infection after outbreaks of gastroenteritis:prospective cohort survey in institutionalised young people. BMJ, 2004,24:204-205.

32 Moreno Y, Piqueres P, Alonso JL, et al. Survival and viability of Helicobacter pylori after inoculation into chlorinated drinking water. Water Res, 2007, 41:3490-3496.

33 Samra ZQ, Javaid U, Ghafoor S, et al. PCR assay targeting virulence 
genes of Helicobacter pylori isolated from drinking water and clinical samples in Lahore metropolitan,Pakistan. J Water Health, 2011, 9: 208-216.

34 Hulten K, Han SW, Enroth H, et al. Helicobacter pylori in the drinking water in Peru. Gastroenterology, 1996, 110:1031-1035.

35 Horiuchi T, Ohkusa T, Watanabe M, et al. Helicobacter pylori DNA in drinking water in Japan. Microbiol Immunol, 2001,45:515-519.

36 Quaglia NC, Dambrosio A, Normanno G, et al. Survival of Helicobacter pylori in artificially contaminated ultrahigh temperature and pasteurized milk. Food Microbiol. 2007, 24:296-300.

37 Cellini L, Del Vecchio A, Di Candia M, et al. Detection of free and plankton -associated Helicobacter pylori in seawater. J Appl Microbiol, 2004, 97:285-292.

38 Queralt N, Bartolomé R, Araujo R. Detection of Helicobacter pylori DNA in human faeces and water with different levels of faecal pollution in the north-east of Spain. J Appl Microbiol, 2005,98:889-895.

39 Cellini L, Di CE, Grande R, et al. Detection of Helicobacter pylori associated with zooplankton. Aquatic Microbial Ecology, 2005,40:115-120.

40 Moreno Y, Ferrús MA. Specific detection of cultivable Helicobacter pylori cells from wastewater treatment plants. Helicobacter, 2012,17:327-332.
41 Chen X, Luo J, Zhou L. Case Study of Helicobacter Pylori Infection in Residents of Buyi Ethnic Group in Luodian County, Guizhou Province . Guizhou Medicine, 2011, 35 (5): 415-417.

42 Zhang L, Zhou Y, Zhang Z, et al. Status of Helicobacter pylori infection in children and adolescents in Wuwei City, Gansu Province and the Characteristics of Its Strains . Chinese Medical Journal, 2009, 89 (38): 2682-2685.

43 Zhang T, Zhang T, Zhao D, etc. Meta-analysis on Correlative Factors of Helicobacter Pylori Infection in Chinese Population. World Chinese Journal of Digestion, 2009, 17 (15): 1582-1589.

44 Papiez D, Konturek PC, Bielanski W, et al. Prevalence ofHelicobacter pylori infection in Polish shepherds and their families.Dig Liver Dis, 2003, 35:10-15.

45 Gisbert JP. The recurrence of Helicobacter pylori infection: incidence and variables influencing it. a critical review . Am J Gastroenterol, 2005, 100: 2083-2099.

46 Niv Y, Hazazi R. Helicobacter pylori recurrence in developed and developing countries: meta-analysis of 13C-urea breath test follow-up after eradication. Helicobacter, 2008, 13:56-61.

47 Ramirez-Ramos A, Gilman RH, Leon -Barua R, et al. Rapid recurrence of Helicobacter pylori infection in Peruvian patients after successful eradication. Clin Infect Dis, 1997, 25:1027-1031. 